\title{
Robust Sliding Mode Control for Discrete Stochastic Systems with Mixed Time-delays, Randomly Occurring Uncertainties and Nonlinearities
}

\author{
Jun Hu, Zidong Wang, Senior Member, IEEE, Huijun Gao, Senior Member, IEEE, and Lampros K. Stergioulas
}

\begin{abstract}
This paper investigates the robust sliding mode control (SMC) problem for a class of uncertain nonlinear stochastic systems with mixed time-delays. Both the sector-like nonlinearities and the norm-bounded uncertainties enter into the system in randomly ways, and such randomly occurring uncertainties (ROUs) and randomly occurring nonlinearities (RONs) obey certain mutually uncorrelated Bernoulli distributed white noise sequences. The mixed time-delays consist of both the discrete and the distributed delays. The time-varying delays are allowed in state. By employing the idea of delay-fractioning and constructing a new Lyapunov-Krasovskii functional, sufficient conditions are established to ensure the stability of the system dynamics in the specified sliding surface by solving certain semidefinite programming problem. A full-state feedback SMC law is designed to guarantee the reaching condition. A simulation example is given to demonstrate the effectiveness of the proposed SMC scheme.
\end{abstract}

Index Terms-Sliding mode control, randomly occurring uncertainties (ROUs), randomly occurring nonlinearities (RONs), discrete time-delays, infinite distributed delays.

\section{INTRODUCTION}

$\mathbf{I}$ $\mathrm{N}$ the past two decades, SMC has become one of the most active branches of control theory that has found successful applications in a variety of engineering systems such as robot manipulators, aircrafts, electrical motors and automotive engines [4], [6], [14], [16]. Also, considerable research attention has been devoted to the theoretical research on SMC problems for different systems. For example, the concept of SMC has been widely employed for uncertain systems [2], [8], [23], stochastic systems [7], [15], [19] as well

Manuscript received July 10, 2010; Accepted for publication August 14, 2011

Copyright (c) 2009 IEEE. Personal use of this material is permitted. However, permission to use this material for any other purposes must be obtained from the IEEE by sending a request to pubs-permissions@ieee.org.

This work was supported in part by the National Natural Science Foundation of China under Grants 61028008, 60825303 and 60834003, National 973 Project under Grant 2009CB320600, the Fok Ying Tung Education Fund under Grant 111064, the Special Fund for the Author of National Excellent Doctoral Dissertation of China under Grant 2007B4, the Key Laboratory of Integrated Automation for the Process Industry (Northeastern University) from the Ministry of Education of China, the Engineering and Physical Sciences Research Council (EPSRC) of the U.K. under Grant GR/S27658/01, the Royal Society of the U.K., and the Alexander von Humboldt Foundation of Germany.

J. Hu and H. Gao are with the Research Institute of Intelligent Control and Systems, Harbin Institute of Technology, Harbin 150001, China.

Z. Wang and L. K. Stergioulas are with the Department of Information Systems and Computing, Brunel University, Uxbridge, Middlesex, UB8 3PH, United Kingdom. (Email: Zidong.Wang@brunel.ac.uk) as fuzzy systems [12], [22]. It should be pointed out that most results mentioned above have been concerned with continuoustime systems. Recently, many important results have been reported on the SMC problem for discrete-time systems, see [1], [5], [10], [20], [21]. In [5], a discrete-time sliding mode reaching condition and the concept of quasi-sliding mode have been thoroughly investigated, which have later been applied in [20], [21] to address the SMC problems for a class of uncertain time-delay systems.

It is well known that time-delays are frequently encountered in many engineering systems. The existence of time delays may cause undesirable dynamic behaviors such as oscillation and instability. According to the occurrence way of timedelays, they can be generally classified into two types: discrete delays and distributed delays. Over the past decades, much effort has been made to address the SMC problem for timedelay systems, see e.g. [11], [20], [21]. It is worth mentioning that most of results are applicable to continuous systems only, and the relevant results for discrete systems with mixed delays (i.e., both discrete and distributed) have been very few. Note that the distributed delays in the discrete-time setting is an emerging concept that has been proposed in [18] for complex networks. Such a situation gives us the initial motivation for establishing a unified framework in order to handle the mixed time-delays for discrete-time systems by using the SMC scheme.

Nonlinearities and uncertainties serve as two important kinds of complexities for system modeling. In the networked world nowadays, the nonlinear disturbances and the parameter uncertainties may be subject to random changes in environmental circumstances, for instance, network-induced random failures and repairs of components, sudden environmental disturbances etc. Therefore, both the nonlinearities and the uncertainties may occur in a probabilistic way with certain types and intensity, which is particularly true in a networked environment. Very recently, in [18], the concept of RONs has been introduced to model the randomly occurring nonlinear functions for complex networks, but ROUs have not yet received adequate research attention. It is, therefore, our aim in this paper to shorten such a gap by applying the delayfractioning approach (see e.g. [13]), for handling the SMC problem with ROUs, RONs and mixed time-delays.

Motivated by the above discussion, we deal with the robust SMC problem for a class of discrete stochastic systems with 
ROUs, RONs and mixed time-delays. By using the delayfractioning approach, a sufficient condition is presented to ensure the stability of the sliding mode dynamics by means of the feasibility of a certain semidefinite programming problem with an equality constraint. A computational algorithm is used to convert the original nonconvex problem into a minimization problem, and an SMC law is synthesized. The main contributions of this paper can be highlighted as follows: (i) the concepts of ROUs, RONs and mixed time-delays are, for the first time, introduced together for the SMC problem in order to reflect a more realistic environment; (ii) the delay-fractioning approach as well as a new Lyapunov-Krasovskii functional is applied, for the first time, to design the SMC law with hope to reduce the possible conservatism caused by the time-delays; and (iii) intensive stochastic analysis is carried out to account for the random nature of the appearance of the uncertainties and nonlinearities.

\section{Problem Formulation and Preliminaries}

Consider the following class of uncertain nonlinear stochastic systems:

$$
\left\{\begin{aligned}
x_{k+1}= & \left(A+\alpha_{k} \Delta A\right) x_{k}+\left(A_{d}+\beta_{k} \Delta A_{d}\right) x_{k-d_{k}} \\
& +B\left(u_{k}+f\left(x_{k}\right)\right)+C \sum_{p=1}^{+\infty} \mu_{p} x_{k-p} \\
& +\sum_{i=1}^{d} \gamma_{k}^{i} D g_{i}\left(x_{k}\right)+E \sigma\left(x_{k}, x_{k-d_{k}}\right) \omega_{k} \\
x_{k}= & \varphi_{k}, \quad \forall k \in \mathbb{Z}^{-}
\end{aligned}\right.
$$

where $x_{k} \in \mathbb{R}^{n}$ is the state vector, $u_{k} \in \mathbb{R}^{q}$ is the control input, $f\left(x_{k}\right)$ denotes the unknown nonlinear function that is bounded in terms of Euclidean norm, i.e., there exists a known scalar function $\rho_{f}\left(x_{k}\right)$ such that $\left\|f\left(x_{k}\right)\right\| \leq \rho_{f}\left(x_{k}\right)$, and $\omega_{k}$ is a one-dimensional, zero-mean Gaussian white noise sequence on a probability space $(\Omega, \mathcal{F}, \mathcal{P})$ with $\mathbb{E}\left\{\omega_{k}^{2}\right\}=1$. $A, B, A_{d}$, $C, D$ and $E$ are known matrices, and $\varphi_{k}$ is a given initial condition.

The nonlinear function $\sigma(\cdot, \cdot)$ satisfies

$$
\sigma^{T}(x, y) \sigma(x, y) \leq \rho_{1} x^{T} x+\rho_{2} y^{T} y, \quad \forall x, y \in \mathbb{R}^{n}
$$

where $\rho_{1}>0$ and $\rho_{2}>0$ are known scalars.

The real-valued matrices $\Delta A$ and $\Delta A_{d}$ represent the normbounded parameter uncertainties of the following structure

$$
\left[\begin{array}{ll}
\Delta A & \Delta A_{d}
\end{array}\right]=\left[\begin{array}{ll}
H_{a} F & H_{d} F
\end{array}\right] N,
$$

where $H_{a}, H_{d}$ and $N$ are known constant matrices, and $F$ is an unknown matrix function satisfying $F^{T} F \leq I$.

The stochastic variables $\alpha_{k} \in \mathbb{R}$ and $\beta_{k} \in \mathbb{R}$ are Bernoulli distributed white sequences taking values on either 0 or 1 with

$$
\begin{array}{ll}
\operatorname{Prob}\left\{\alpha_{k}=1\right\}=\alpha, & \operatorname{Prob}\left\{\alpha_{k}=0\right\}=1-\alpha, \\
\operatorname{Prob}\left\{\beta_{k}=1\right\}=\beta, & \operatorname{Prob}\left\{\beta_{k}=0\right\}=1-\beta,
\end{array}
$$

where $\alpha \in[0,1]$ and $\beta \in[0,1]$ are known constants.

For each $1 \leq i \leq d$, the nonlinear function $g_{i}: \mathbb{R}^{n} \rightarrow \mathbb{R}^{n}$ stands for the mismatched external nonlinearity that satisfies the following sector-bounded condition:

$$
\left[g_{i}(x)-F_{1 i} x\right]^{T}\left[g_{i}(x)-F_{2 i} x\right] \leq 0, \quad \forall x \in \mathbb{R}^{n}
$$

where $F_{1 i}$ and $F_{2 i}$ are known real constant matrices, and $F_{i}=$ $F_{1 i}-F_{2 i}$ is symmetric positive definite matrix.

The stochastic variables $\gamma_{k}^{i} \in \mathbb{R}(1 \leq i \leq d)$, which account for the phenomena of multiple RONs, are another series of Bernoulli distributed white sequences taking values on 0 or 1 with

$$
\operatorname{Prob}\left\{\gamma_{k}^{i}=1\right\}=\gamma^{i}, \quad \operatorname{Prob}\left\{\gamma_{k}^{i}=0\right\}=1-\gamma^{i},
$$

where $\gamma^{i} \in[0,1]$ are known constants. Furthermore, the constant $\mu_{p} \geq 0(p=1,2, \ldots)$ satisfies the following convergence condition

$$
\bar{\mu}=\sum_{p=1}^{\infty} \mu_{p} \leq \sum_{p=1}^{\infty} p \mu_{p}<+\infty .
$$

Remark 1: The random variables $\alpha_{k}$ and $\beta_{k}$ are introduced to characterize the phenomenon of the ROUs. Such a description is more suitable for reflecting parameter variations of a random nature especially in the network transmission. On the other hand, it is customary that the sector-like description of the nonlinearity $g_{i}$ in (5) is said to belong to sectors $\left[F_{1 i}, F_{2 i}\right]$ [9], which is more general than the usually used Lipschitztype functions, see e.g. [17], [18]. By employing such a description, it would be possible to reduce the conservatism of the results caused by quantifying the nonlinearities via the convex optimization technique.

Remark 2: In this paper, the random variables $\gamma_{k}^{i}(i=$ $1,2, \ldots, d)$ are used to model the probability distribution of the nonlinear functions in system (1). Together with condition (5), each $g_{i}$ enters into the system in a random way according to an individual Bernoulli distribution. This description can reflect the fact that the multiple RONs can appear or disappear in a probabilistic way due to unpredictable changes of the environmental circumstances.

Before proceeding, we make the following assumptions.

Assumption 1: The parameter uncertainties $\Delta A$ and $\Delta A_{d}$ are bounded in terms of Euclidean norm.

Assumption 2: The positive integer $d_{k}$ describes the discrete time-varying delay that satisfies:

$$
d_{m} \leq d_{k} \leq d_{M}
$$

where $d_{m}$ and $d_{M}$ are known positive integers representing the lower and upper bounds of the time-delay, respectively. The lower bound of delay $d_{m}$ can always be described by $d_{m}=\tau m$, where $\tau$ and $m$ are integers.

Assumption 3: a) The stochastic variables $\alpha_{k}, \beta_{k}$ and $\gamma_{k}^{i}$ are mutually uncorrelated random variables which are also unrelated with $\omega_{k}$. b) The stochastic variables $\alpha_{k}, \beta_{k}$ and $\gamma_{k}^{i}$ $(i=1,2, \ldots, d)$ are independent of $\mathcal{F}$.

\section{DESIGN OF SMC}

In this section, a sufficient condition is presented to ensure the stability of sliding mode dynamics, and an SMC law is synthesized to drive the state trajectories of system (1) onto the pre-specified sliding surface. 


\section{A. Sliding surface}

Firstly, a discrete-time switching function is constructed as follows:

$$
s_{k}=G x_{k}-G A x_{k-1}
$$

where $G$ is to be designed such that $G B$ is nonsingular and $G \mathfrak{B}=0$, where $\mathfrak{B}=\left[\begin{array}{lll}C & D & E\end{array}\right]$. In this paper, we choose $G=B^{T} P$ with $P>0$ to confirm the nonsingularity of $G B$, and $B$ is assumed to be of full column rank.

When the state trajectories of the system (1) enter into the ideal quasi-sliding mode $s_{k+1}=s_{k}=0$, the equivalent control law of the sliding motion can be obtained,

$$
u_{k}^{e q}=-(G B)^{-1} G \mathscr{A}_{k}-f\left(x_{k}\right)
$$

where $\mathscr{A}_{k}=\alpha \Delta A x_{k}+\left(A_{d}+\beta \Delta A_{d}\right) x_{k-d_{k}}$.

Substituting (10) as $u_{k}$ into (1), we obtain the sliding mode dynamics as follows:

$$
\left\{\begin{aligned}
x_{k+1}= & \mathcal{A}_{k}-B(G B)^{-1} G \mathscr{A}_{k}+\left(\alpha_{k}-\alpha\right) \Delta A x_{k} \\
& +\left(\beta_{k}-\beta\right) \Delta A_{d} x_{k-d_{k}}+C \sum_{p=1}^{+\infty} \mu_{p} x_{k-p} \\
& +\sum_{i=1}^{d} \gamma_{k}^{i} D g_{i}\left(x_{k}\right)+E \sigma\left(x_{k}, x_{k-d_{k}}\right) \omega_{k},
\end{aligned}\right.
$$

where $\mathcal{A}_{k}=(A+\alpha \Delta A) x_{k}+\left(A_{d}+\beta \Delta A_{d}\right) x_{k-d_{k}}$.

\section{B. Performance of the sliding motion}

Before proceeding, we introduce the following lemmas.

Lemma 1: For any real vectors $a, b$ and matrix $P>0$ of appropriate dimensions, the following inequality holds

$$
a^{T} b+b^{T} a \leq a^{T} P a+b^{T} P^{-1} b .
$$

Lemma 2: Let $Q=Q^{T}, N$ and $H$ be real matrices of compatible dimensions with $F$ satisfying $F^{T} F \leq I$. Then $Q+N F H+H^{T} F^{T} N^{T}<0$ if and only if there exists a scalar $\varepsilon>0$ such that $Q+\varepsilon N N^{T}+\varepsilon^{-1} H^{T} H<0$ or, equivalently

$$
\left[\begin{array}{ccc}
Q & \varepsilon N & H^{T} \\
* & -\varepsilon I & 0 \\
* & * & -\varepsilon I
\end{array}\right]<0 .
$$

The following theorem gives a sufficient condition to guarantee the robustly asymptotic mean-square stability of the sliding mode dynamics (11).

Theorem 1: Consider the system (11) and the sliding surface described by (9). For a given scalar $\varrho \in(0,1)$, the sliding mode dynamics (11) is robustly asymptotically mean-square stable if there exist matrices $P>0, Q>0, R>0, S_{1}>0$, $S_{2}>0, T>0$, real matrices $\mathcal{X}, \mathcal{Y}, \mathcal{Z}$, and scalars $\lambda^{*}>0$, $\varepsilon>0, \varphi>0$ satisfying

$$
\left[\begin{array}{cccccc}
\Phi_{11} & \Phi_{12} & \vartheta_{1} \mathcal{X} & \vartheta_{2} \mathcal{Y} & \Phi_{15} & \varepsilon \Phi_{16}^{T} \\
* & \Phi_{22} & 0 & 0 & \Phi_{25} & 0 \\
* & * & -\varrho P & 0 & 0 & 0 \\
* & * & * & -\varrho P & 0 & 0 \\
* & * & * & * & -\varepsilon I & 0 \\
* & * & * & * & * & -\varepsilon I
\end{array}\right]<0
$$

$$
\begin{aligned}
& {\left[\begin{array}{cccccc}
\Phi_{11} & \Phi_{12} & \vartheta_{1} \mathcal{X} & \vartheta_{2} \mathcal{Z} & \Phi_{15} & \varepsilon \Phi_{16}^{T} \\
* & \Phi_{22} & 0 & 0 & \Phi_{25} & 0 \\
* & * & -\varrho P & 0 & 0 & 0 \\
* & * & * & -\varrho P & 0 & 0 \\
* & * & * & * & -\varepsilon I & 0 \\
* & * & * & * & * & -\varepsilon I
\end{array}\right]<0} \\
& E^{T} P E \leq \lambda^{*} I, \\
& B^{T} P \mathfrak{B}=0,
\end{aligned}
$$

where

$$
\begin{aligned}
& \Phi_{11}=\operatorname{sym}\left(\Pi_{1}+\hat{h}^{2} \sum_{i=1}^{d} r_{i} \Xi_{1}^{T} P D \Xi_{g i}+\hat{h}^{2} \Xi_{1}^{T} P C \Xi_{g \mu}\right) \\
& +\Pi_{2}+\Pi_{3}, \\
& \Phi_{12}=\left[\begin{array}{llll}
\sqrt{2} \hat{h} \Xi_{1}^{T} P & 2 \hat{h} \Xi_{2}^{T} P B & 0 & 0
\end{array}\right], \\
& \Phi_{22}=\operatorname{diag}\left\{-P,-B^{T} P B,-P,-P\right\}, \\
& \Phi_{15}^{T}=\left[\begin{array}{llll}
0 & r_{1} \hat{h}^{2} \alpha H_{a}^{T} P D & r_{2} \hat{h}^{2} \alpha H_{a}^{T} P D & \ldots \\
0 & r_{1} \hat{h}^{2} \beta H_{d}^{T} P D & r_{2} \hat{h}^{2} \beta H_{d}^{T} P D & \ldots
\end{array}\right. \\
& \left.r_{d} \hat{h}^{2} \alpha H_{a}^{T} P D \quad \hat{h}^{2} \alpha H_{a}^{T} P C\right] \\
& \left.r_{d} \hat{h}^{2} \beta H_{d}^{T} P D \quad \hat{h}^{2} \beta H_{d}^{T} P C\right], \\
& \Phi_{16}=\left[\begin{array}{lllll}
N & 0_{n_{N} \times m n} & 0_{n_{N} \times n} & 0_{n_{N} \times(d+2) n} \\
\hline 0_{n_{N} \times n} & 0_{n_{N} \times m n} & N & 0_{n_{N} \times(d+2) n}
\end{array}\right], \\
& \Phi_{25}^{T}=\left[\begin{array}{ll}
\mathscr{X}_{11} & \mathscr{X}_{12}
\end{array}\right] \text {, } \\
& \mathscr{X}_{11}=\left[\begin{array}{cc}
\sqrt{2} \hat{h} \alpha H_{a}^{T} P & 2 \hat{h} \alpha H_{a}^{T} P B \\
\sqrt{2} \hat{h} \beta H_{d}^{T} P & 2 \hat{h} \beta H_{d}^{T} P B
\end{array}\right], \\
& \mathscr{X}_{12}=\left[\begin{array}{cc}
\sqrt{\bar{\alpha}} \hat{h} H_{a}^{T} P & 0 \\
0 & \sqrt{\bar{\beta}} \hat{h} H_{d}^{T} P
\end{array}\right], \\
& \Xi_{1}=\left[\begin{array}{llll}
A & 0_{n \times m n} & A_{d} & 0_{n \times(d+2) n}
\end{array}\right], \\
& \Xi_{2}=\left[\begin{array}{lll}
0_{n \times(m+1) n} & A_{d} & 0_{n \times(d+2) n}
\end{array}\right], \\
& \Xi_{g i}=\left[\begin{array}{llll}
0_{n \times(m+i+2) n} & I_{n \times n} & 0_{n \times(d-i) n} & 0_{n \times n}
\end{array}\right] \text {, } \\
& \Xi_{g \mu}=\left[\begin{array}{ll}
0_{n \times(m+d+3) n} & I_{n \times n}
\end{array}\right] \text {, } \\
& \Pi_{1}=\left[\begin{array}{lll}
\mathcal{X} & \mathcal{Y} & \mathcal{Z}
\end{array}\right] \\
& \times\left[\begin{array}{cccc}
I_{n \times n} & 0_{n \times(m+1) n} & -I_{n \times n} & 0_{n \times(d+1) n} \\
\hline 0_{n \times m n} & I_{n \times n} & -I_{n \times n} & 0_{n \times(d+2) n} \\
\hline 0_{n \times(m+1) n} & I_{n \times n} & -I_{n \times n} & 0_{n \times(d+1) n}
\end{array}\right], \\
& \Pi_{2}=W_{R}^{T} \mathcal{R} W_{R}, \quad \mathcal{R}=\operatorname{diag}\{R,-R\}, \quad \Pi_{3}=W_{\Theta}^{T} \Theta W_{\Theta},
\end{aligned}
$$

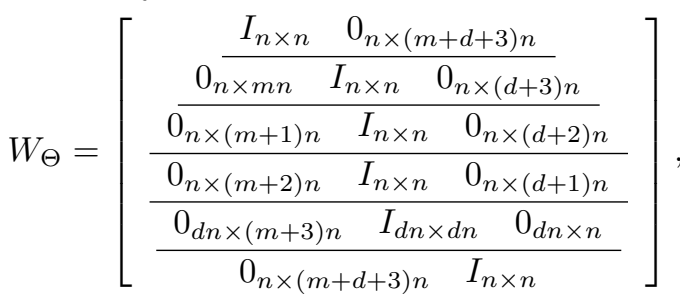

$$
\begin{aligned}
& W_{R}=\left[\begin{array}{ccc}
I_{m n \times m n} & 0_{m n \times(d+4) n} \\
0_{m n \times n} & I_{m n \times m n} & 0_{m n \times(d+3) n}
\end{array}\right] \text {, } \\
& \Theta=\left[\begin{array}{cc}
\aleph_{11} & \aleph_{12} \\
* & \aleph_{22}
\end{array}\right] \text {, } \\
& \aleph_{11}=\operatorname{diag}\left\{\Theta_{11},-S_{1}, \hat{h}^{2} \lambda^{*} \rho_{2} I-Q,-S_{2}\right\} \text {, } \\
& \aleph_{12}^{T}=\left[\begin{array}{cccc}
\Theta_{15}^{T} & 0 & 0 & 0 \\
0 & 0 & 0 & 0
\end{array}\right] \text {, }
\end{aligned}
$$




$$
\begin{aligned}
& \aleph_{22}=\left[\begin{array}{cc}
\Theta_{55} & \hat{h}^{2} \Theta_{56} \\
* & 2 \hat{h}^{2} C^{T} P C-\frac{1}{\bar{\mu}} T
\end{array}\right], \\
& \Theta_{11}=(2 \varrho \hbar-1) P+\hat{h}^{2} \lambda^{*} \rho_{1} I+\left(d_{M}-d_{m}+1\right) Q \\
& +S_{1}+S_{2}+\bar{\mu} T-\varphi \sum_{i=1}^{d} \tilde{F}_{i}, \\
& \Theta_{15}=\left[\begin{array}{llll}
\varphi \bar{F}_{1} & \varphi \bar{F}_{2} & \cdots & \varphi \bar{F}_{d}
\end{array}\right], \\
& \Theta_{55}=\left[\Theta_{55}^{(i j)}\right], \quad(i, j=1,2, \ldots, d) \\
& \Theta_{55}^{(i i)}=\hat{h}^{2}\left(\left(\gamma^{i}\right)^{2}+\gamma^{i}\right) D^{T} P D-2 \varphi I, \\
& \Theta_{55}^{(i j)}=2 \gamma^{i} \gamma_{j} \hat{h}^{2} D^{T} P D,(i \neq j) \\
& \Theta_{56}^{T}=\left[\begin{array}{llll}
r_{1} I & r_{2} I & \ldots & r_{d} I
\end{array}\right] C^{T} P D, \\
& \tilde{F}_{i}=F_{1 i}^{T} F_{2 i}+F_{2 i}^{T} F_{1 i}, \bar{F}_{i}=F_{1 i}^{T}+F_{2 i}^{T} \text {, } \\
& \hbar=2 d_{M}-d_{m}, \hat{h}=\sqrt{1+2 \varrho \hbar}, \quad \vartheta_{1}=\sqrt{d_{M}}, \\
& \vartheta_{2}=\sqrt{d_{M}-d_{m}}, \quad \bar{\alpha}=\alpha(1-\alpha), \quad \bar{\beta}=\beta(1-\beta)
\end{aligned}
$$

and $n_{N}$ is the number of row in matrix $N \cdot \operatorname{sym}(\Im)$ represents $\Im+\Im^{T}$.

Proof: Choose the following Lyapunov-Krasovskii functional candidate for system (11):

$$
V_{k}=\sum_{i=1}^{6} V_{k}^{i}
$$

where

$$
\begin{aligned}
V_{k}^{1} & =x_{k}^{T} P x_{k} \\
V_{k}^{2} & =\sum_{l=k-d_{k}}^{k-1} x_{l}^{T} Q x_{l}+\sum_{j=-d_{M}+1}^{-\tau m} \sum_{l=k+j}^{k-1} x_{l}^{T} Q x_{l} \\
V_{k}^{3} & =\sum_{l=k-d_{m}}^{k-1} x^{T}(l) S_{l} x_{l}+\sum_{l=k-d_{M}}^{k-1} x_{l}^{T} S_{2} x_{l} \\
V_{k}^{4} & =\sum_{l=k-\tau}^{k-1} \Gamma_{l}^{T} R \Gamma_{l} \\
V_{k}^{5} & =\varrho \sum_{j=-d_{M}+1}^{0} \sum_{l=k-1+j}^{k-1} \eta_{l}^{T} P \eta_{l}+\varrho \sum_{j=-d_{M}+1}^{-\tau m} \sum_{l=k-1+j}^{k-1} \eta_{l}^{T} P \eta_{l} \\
V_{k}^{6} & =\sum_{p=1}^{+\infty} \mu_{p} \sum_{l=k-p}^{k-1} x_{l}^{T} T x_{l} \\
\eta_{l} & =x_{l+1}-x_{l}, \Gamma_{l}=\operatorname{col}\left\{x_{l}, x_{l-\tau}, \ldots, x_{l-(m-1) \tau}\right\}
\end{aligned}
$$

with $P>0, Q>0, R>0, S_{1}>0, S_{2}>0, T>0$ being matrices to be determined. Here, $\operatorname{col}\{\cdots\}$ denotes a vector column with blocks given by the vectors in $\{\cdots\}$.

By using Lemma 1 and noting Assumption 3, we have

$$
\begin{aligned}
& \mathbb{E}\left\{\Delta V_{k}^{1}\right\} \\
\leq & \mathbb{E}\left\{\xi _ { k } ^ { T } \left[2 \hat{\Xi}_{1}^{T} P \hat{\Xi}_{1}+2 \hat{\Xi}_{1}^{T} P C \Xi_{g \mu}+2 \sum_{i=1}^{d} r_{i} \hat{\Xi}_{1}^{T} P D \Xi_{g i}\right.\right. \\
& +4 \hat{\Xi}_{2}^{T} G^{T}(G B)^{-1} G \hat{\Xi}_{2}+2 \Xi_{g \mu}^{T} C^{T} P C \Xi_{g \mu}
\end{aligned}
$$

$$
\begin{aligned}
& \left.+2 \sum_{i=1}^{d} r_{i} \Xi_{g \mu}^{T} C^{T} P D \Xi_{g i}+\bar{\alpha} \hat{\Xi}_{3}^{T} P \hat{\Xi}_{3}+\bar{\beta} \hat{\Xi}_{4}^{T} P \hat{\Xi}_{4}\right] \xi_{k} \\
& +2\left[\sum_{i=1}^{d} \gamma^{i} D g_{i}\left(x_{k}\right)\right]^{T} P\left[\sum_{i=1}^{d} \gamma^{i} D g_{i}\left(x_{k}\right)\right] \\
& +\sum_{i=1}^{d} \gamma^{i}\left(1-\gamma^{i}\right) g_{i}^{T}\left(x_{k}\right) D^{T} P D g_{i}\left(x_{k}\right) \\
& \left.+\sigma^{T}\left(x_{k}, x_{k-d_{k}}\right) E^{T} P E \sigma\left(x_{k}, x_{k-d_{k}}\right)-x_{k}^{T} P x_{k}\right\}
\end{aligned}
$$

where

$$
\begin{aligned}
& \xi_{k}^{T}=\left[\begin{array}{lllll}
\Gamma_{k}^{T} & x_{k-d_{m}}^{T} & x_{k-d_{k}}^{T} & x_{k-d_{M}}^{T} & g^{T}\left(x_{k}\right) \\
& & \sum_{p=1}^{+\infty} \mu_{p} x_{k-p}^{T}
\end{array}\right], \\
& g\left(x_{k}\right)=\left[\begin{array}{llll}
g_{1}^{T}\left(x_{k}\right) & g_{2}^{T}\left(x_{k}\right) & \cdots & g_{d}^{T}\left(x_{k}\right)
\end{array}\right]^{T}, \\
& \hat{\Xi}_{1}=\left[\begin{array}{llll}
A+\alpha \Delta A & 0_{n \times m n} & A_{d}+\beta \Delta A_{d} & 0_{n \times(d+2) n}
\end{array}\right], \\
& \hat{\Xi}_{2}=\left[\begin{array}{llll}
\alpha \Delta A & 0_{n \times m n} & A_{d}+\beta \Delta A_{d} & 0_{n \times(d+2) n}
\end{array}\right], \\
& \hat{\Xi}_{3}=\left[\begin{array}{llll}
\Delta A & 0_{n \times(m+d+3) n}
\end{array}\right], \\
& \hat{\Xi}_{4}=\left[\begin{array}{lll}
0_{n \times(m+1) n} & \Delta A_{d} & 0_{n \times(d+2) n}
\end{array}\right],
\end{aligned}
$$

and $\bar{\alpha}, \bar{\beta}, \Xi_{g \mu}, \Xi_{g i}$ are defined in Theorem 1 (below (17)).

On the other hand, it can be derived that

$$
\begin{aligned}
& \sum_{i=2}^{6} \mathbb{E}\left\{\Delta V_{k}^{i}\right\} \\
\leq & \mathbb{E}\left\{x_{k}^{T}\left(\left(d_{M}-d_{m}+1\right) Q+S_{1}+S_{2}+\bar{\mu} T\right) x_{k}\right. \\
& -x_{k-d_{k}}^{T} Q x_{k-d_{k}}-x_{k-d_{m}}^{T} S_{1} x_{k-d_{m}}-x_{k-d_{M}}^{T} S_{2} x_{k-d_{M}} \\
+ & \Gamma_{k}^{T} R \Gamma_{k}-\Gamma_{k-\tau}^{T} R \Gamma_{k-\tau}+2 \varrho \hbar x_{k+1}^{T} P x_{k+1} \\
+ & 2 \varrho \hbar x_{k}^{T} P x_{k}-\sum_{l=k-d_{M}}^{k-1} \eta_{l}^{T} \varrho P \eta_{l}-\sum_{l=k-d_{k}}^{k-\tau m-1} \eta_{l}^{T} \varrho P \eta_{l} \\
& -\sum_{l=k-d_{M}}^{k-d_{k}-1} \eta_{l}^{T} \varrho P \eta_{l}-\frac{1}{\bar{\mu}}\left[\sum_{p=1}^{+\infty} \mu_{p} x_{k-p}\right]^{T} \\
& \left.\times T\left[\sum_{p=1}^{+\infty} \mu_{p} x_{k-p}\right]\right\}
\end{aligned}
$$

It follows from (2) and (16) that

$$
\begin{aligned}
& \sigma^{T}\left(x_{k}, x_{k-d_{k}}\right) E^{T} P E \sigma\left(x_{k}, x_{k-d_{k}}\right) \\
\leq & \lambda^{*}\left(\rho_{1} x_{k}^{T} x_{k}+\rho_{2} x_{k-d_{k}}^{T} x_{k-d_{k}}\right) .
\end{aligned}
$$

For each $1 \leq i \leq d,(5)$ is equivalent to

$$
\left[\begin{array}{c}
x_{k} \\
g_{i}\left(x_{k}\right)
\end{array}\right]^{T}\left[\begin{array}{cc}
-\tilde{F}_{i} & \bar{F}_{i} \\
* & -2 I
\end{array}\right]\left[\begin{array}{c}
x_{k} \\
g_{i}\left(x_{k}\right)
\end{array}\right] \geq 0
$$

where matrices $\tilde{F}_{i}$ and $\bar{F}_{i}$ are defined in Theorem 1 (below (17)). Furthermore, according to the definition of $\eta_{l}$, for any matrices $\mathcal{X}, \mathcal{Y}, \mathcal{Z}$ with appropriate dimensions, the following 
equations always hold:

$$
\begin{aligned}
& 0=2 \xi_{k}^{T} \mathcal{X}\left[x_{k}-x_{k-d_{M}}-\sum_{l=k-d_{M}}^{k-1} \eta_{l}\right], \\
& 0=2 \xi_{k}^{T} \mathcal{Y}\left[x_{k-\tau m}-x_{k-d_{k}}-\sum_{l=k-d_{k}}^{k-\tau m-1} \eta_{l}\right], \\
& 0=2 \xi_{k}^{T} \mathcal{Z}\left[x_{k-d_{k}}-x_{k-d_{M}}-\sum_{l=k-d_{M}}^{k-d_{k}-1} \eta_{l}\right] .
\end{aligned}
$$

Noting (19)-(25), we have

$$
\begin{aligned}
& \mathbb{E}\left\{\Delta V_{k}\right\} \\
\leq & \mathbb{E}\left\{\xi _ { k } ^ { T } \left[( \frac { d _ { k } - d _ { m } } { d _ { M } - d _ { m } } ) \left(\Pi_{1}+\Pi_{1}^{T}+\Pi_{2}+\Pi_{3}\right.\right.\right. \\
& +2 \hat{h}^{2} \hat{\Xi}_{1}^{T} P \hat{\Xi}_{1}+2 \hat{h}^{2} \sum_{i=1}^{d} r_{i} \hat{\Xi}_{1}^{T} P D \Xi_{g i}+2 \hat{h}^{2} \hat{\Xi}_{1}^{T} P C \Xi_{g \mu} \\
& +4 \hat{h}^{2} \hat{\Xi}_{2}^{T} G^{T}(G B)^{-1} G \hat{\Xi}_{2}+\bar{\alpha} \hat{h}^{2} \hat{\Xi}_{3}^{T} P \hat{\Xi}_{3}+\bar{\beta} \hat{h}^{2} \hat{\Xi}_{4}^{T} P \hat{\Xi}_{4} \\
& \left.+d_{M} \mathcal{X}(\varrho P)^{-1} \mathcal{X}^{T}+\left(d_{M}-d_{m}\right) \mathcal{Y}(\varrho P)^{-1} \mathcal{Y}^{T}\right) \\
& +\left(\frac{d_{M}-d_{k}}{d_{M}-d_{m}}\right)\left(\Pi_{1}+\Pi_{1}^{T}+\Pi_{2}+\Pi_{3}+2 \hat{h}^{2} \hat{\Xi}_{1}^{T} P \hat{\Xi}_{1}\right. \\
& +2 \hat{h}^{2} \sum_{i=1}^{d} r_{i} \hat{\Xi}_{1}^{T} P D \Xi_{g i}+2 \hat{h}^{2} \hat{\Xi}_{1}^{T} P C \Xi_{g \mu}+4 \hat{h}^{2} \hat{\Xi}_{2}^{T} G^{T} \\
& \times(G B)^{-1} G \hat{\Xi}_{2}+\bar{\alpha} \hat{h}^{2} \hat{\Xi}_{3}^{T} P \hat{\Xi}_{3}+\bar{\beta} \hat{h}^{2} \hat{\Xi}_{4}^{T} P \hat{\Xi}_{4} \\
& \left.\left.\left.+d_{M} \mathcal{X}(\varrho P)^{-1} \mathcal{X} T+\left(d_{M}-d_{m}\right) \mathcal{Z}(\varrho P)^{-1} \mathcal{Z}^{T}\right)\right] \xi_{k}\right\}
\end{aligned}
$$

where

$$
\begin{aligned}
& \Sigma_{1}=\left[\varrho P \eta_{l}+\mathcal{X}^{T} \xi_{k}\right]^{T}(\varrho P)^{-1}\left[\varrho P \eta_{l}+\mathcal{X}^{T} \xi_{k}\right] \\
& \Sigma_{2}=\left[\varrho P \eta_{l}+\mathcal{Y}^{T} \xi_{k}\right]^{T}(\varrho P)^{-1}\left[\varrho P \eta_{l}+\mathcal{Y}^{T} \xi_{k}\right] \\
& \Sigma_{3}=\left[\varrho P \eta_{l}+\mathcal{Z}^{T} \xi_{k}\right]^{T}(\varrho P)^{-1}\left[\varrho P \eta_{l}+\mathcal{Z}^{T} \xi_{k}\right]
\end{aligned}
$$

and $\Pi_{1}, \Pi_{2}$ and $\Pi_{3}$ are defined in Theorem 1 (below (17)).

By using Lemma 2 and applying Schur complement, it follows from (14) and (15) that $\mathbb{E}\left\{\Delta V_{k}\right\}<0$ holds, and therefore the mean-square asymptotic stability of the sliding mode dynamics (11) can be confirmed. This completes the proof.

Remark 3: Comparing with the conventional LyapunovKrasovskii functional for delay systems, (18) exhibits two extra terms, $V_{k}^{4}$ and $V_{k}^{5}$, both of which exists for particular reasons. Specifically, $V_{k}^{4}$ is adjusted to reduce the conservatism in response to the need of delay-fractioning for discrete systems, and $V_{k}^{5}$ is there to fit the SMC framework where a so-called "weighting" scalar parameter $\varrho \in(0,1)$ is enforced. Such a parameter is exploited to reflect both the delay-fractioning approach and parameter uncertainties, and its value can be determined a priori to help the feasibility study of (14)-(17) in Theorem 1.

\section{Computational Algorithm}

Note that there exists an equality constraint condition (17) $B^{T} P \mathfrak{B}=0$ in Theorem 1. Based on the algorithm presented in [11], this constraint can be equivalently converted into $\operatorname{tr}\left[\left(B^{T} P \mathfrak{B}\right)^{T} B^{T} P \mathfrak{B}\right]=0$. By introducing an inequality $\left(B^{T} P \mathfrak{B}\right)^{T} B^{T} P \mathfrak{B} \leq \gamma I(\gamma>0$ is a sufficiently small scalar $)$ and using Schur Complement, we have

$$
\left[\begin{array}{cc}
-\gamma I & \mathfrak{B}^{T} P B \\
B^{T} P \mathfrak{B} & -I
\end{array}\right] \leq 0
$$

Hence, the original nonconvex feasibility problem is now converted into a problem of finding the global solution to the following minimization problem:

$\min \gamma$

subject to (14)-(16) and (27).

\section{Reachability Analysis}

In this part, an SMC law is proposed and the reachability analysis of the specified sliding surface is conducted.

By considering the inequality reaching condition in [5],

$$
\begin{cases}\Delta s_{k} \leq-\kappa U \operatorname{sgn}\left[s_{k}\right]-\kappa V s_{k}, & \text { if } s_{k}>0 \\ \Delta s_{k} \geq-\kappa U \operatorname{sgn}\left[s_{k}\right]-\kappa V s_{k}, & \text { if } s_{k}<0\end{cases}
$$

where $\kappa$ denotes the sampling period, $U=\operatorname{diag}\left\{\mu_{1}, \mu_{2}, \ldots\right.$, $\left.\mu_{q}\right\} \in \mathbb{R}^{q \times q}, V=\operatorname{diag}\left\{\nu_{1}, \nu_{2}, \ldots, \nu_{q}\right\} \in \mathbb{R}^{q \times q}$, and $\mu_{i}>0$, $\nu_{i}>0$ are properly chosen constants satisfying $0<1-\kappa \nu_{i}<$ $1(i=1,2, \ldots, q)$.

Notice that $\Delta A, \Delta A_{d}$ and $f\left(x_{k}\right)$ are all bounded in terms of Euclidean norm, let $\Delta_{a}(k):=\alpha_{k} G \Delta A x_{k}, \Delta_{d}(k):=G\left(A_{d}+\right.$ $\left.\beta_{k} \Delta A_{d}\right) x_{k-d_{k}}$ and $\Delta_{f}(k):=G B f\left(x_{k}\right)$, then there exist $\underline{\delta}_{a}^{i}$, $\bar{\delta}_{a}^{i}, \underline{\delta}_{d}^{i}, \bar{\delta}_{d}^{i}, \underline{\delta}_{f}^{i}$ and $\bar{\delta}_{f}^{i}(i=1,2, \cdots, q)$ satisfying

$$
\underline{\delta}_{a}^{i} \leq \delta_{a}^{i}(k) \leq \bar{\delta}_{a}^{i}, \quad \underline{\delta}_{d}^{i} \leq \delta_{d}^{i}(k) \leq \bar{\delta}_{d}^{i}, \quad \underline{\delta}_{f}^{i} \leq \delta_{f}^{i}(k) \leq \bar{\delta}_{f}^{i}
$$

where $\delta_{a}^{i}(k), \delta_{d}^{i}(k)$ and $\delta_{f}^{i}(k)$ are the $i$ th elements in $\Delta_{a}(k)$, $\Delta_{d}(k)$ and $\Delta_{f}(k)$, respectively. Then, by defining

$$
\begin{aligned}
& \widehat{\Delta}_{a}=\left[\begin{array}{llll}
\widehat{\delta}_{a}^{1} & \widehat{\delta}_{a}^{2} & \cdots & \widehat{\delta}_{a}^{q}
\end{array}\right]^{T}, \quad \widehat{\delta}_{a}^{i}=\frac{\bar{\delta}_{a}^{i}+\underline{\delta}_{a}^{i}}{2}, \\
& \widetilde{\Delta}_{a}=\operatorname{diag}\left\{\widetilde{\delta}_{a}^{1}, \widetilde{\delta}_{a}^{2}, \ldots, \widetilde{\delta}_{a}^{q}\right\}, \quad \widetilde{\delta}_{a}^{i}=\frac{\bar{\delta}_{a}^{i}-\underline{\delta}_{a}^{i}}{2}, \\
& \widehat{\Delta}_{d}=\left[\begin{array}{llll}
\widehat{\delta}_{d}^{1} & \widehat{\delta}_{d}^{2} & \cdots & \widehat{\delta}_{d}^{q}
\end{array}\right]^{T}, \quad \widehat{\delta}_{d}^{i}=\frac{\bar{\delta}_{d}^{i}+\underline{\delta}_{d}^{i}}{2}, \\
& \widetilde{\Delta}_{d}=\operatorname{diag}\left\{\widetilde{\delta}_{d}^{1}, \widetilde{\delta}_{d}^{2}, \ldots, \widetilde{\delta}_{d}^{q}\right\}, \quad \widetilde{\delta}_{d}^{i}=\frac{\bar{\delta}_{d}^{i}-\underline{\delta}_{d}^{i}}{2}, \\
& \widehat{\Delta}_{f}=\left[\begin{array}{llll}
\widehat{\delta}_{f}^{1} & \widehat{\delta}_{f}^{2} & \ldots & \widehat{\delta}_{f}^{q}
\end{array}\right]^{T}, \quad \widehat{\delta}_{f}^{i}=\frac{\bar{\delta}_{f}^{i}+\underline{\delta}_{f}^{i}}{2}, \\
& \widetilde{\Delta}_{f}=\operatorname{diag}\left\{\widetilde{\delta}_{f}^{1}, \widetilde{\delta}_{f}^{2}, \ldots, \widetilde{\delta}_{f}^{q}\right\}, \quad \widetilde{\delta}_{f}^{i}=\frac{\bar{\delta}_{f}^{i}-\underline{\delta}_{f}^{i}}{2},
\end{aligned}
$$

we are now ready to present the design scheme of the robust SMC law.

Theorem 2: Assume that the minimization problem (28) is solvable. For the system (1) with sliding surface (9), where 
$G=B^{T} P$ and $P$ is the solution of (28), if the SMC law is given as follows

$$
\begin{aligned}
u(k)= & -(G B)^{-1}\left(\kappa U \operatorname{sgn}\left[s_{k}\right]+\kappa V s_{k}-s_{k}+\left(\widehat{\Delta}_{a}\right.\right. \\
& \left.\left.+\widehat{\Delta}_{d}+\widehat{\Delta}_{f}\right)+\left(\widetilde{\Delta}_{a}+\widetilde{\Delta}_{d}+\widetilde{\Delta}_{f}\right) \operatorname{sgn}\left[s_{k}\right]\right),
\end{aligned}
$$

then the discrete-time sliding mode reaching condition of system (1) with specified sliding surface (9) is satisfied.

Proof: Together with (32) and (9), we have

$$
\begin{aligned}
\Delta s_{k}= & -\kappa U \operatorname{sgn}\left[s_{k}\right]-\kappa V s_{k}+\Delta_{a}(k)-\left(\widehat{\Delta}_{a}+\widetilde{\Delta}_{a} \operatorname{sgn}\left[s_{k}\right]\right) \\
& +\Delta_{d}(k)-\left(\widehat{\Delta}_{d}+\widetilde{\Delta}_{d} \operatorname{sgn}\left[s_{k}\right]\right) \\
& +\Delta_{f}(k)-\left(\widehat{\Delta}_{f}+\widetilde{\Delta}_{f} \operatorname{sgn}\left[s_{k}\right]\right) .
\end{aligned}
$$

It follows easily from (31) that (29) holds, and then proof of this theorem is complete.

Remark 4: In the stochastic model presented in this paper, there are four main aspects that complicate the design of SMC, i.e., ROUs, RONs, infinite distributed delays as well as the delay-fractioning approach. In our main results, all these four aspects have been explicitly reflected, where the occurrence probabilities $\alpha$ and $\beta$ are there for the ROUs, the occurrence probabilities $\gamma^{i}(i=1,2, \ldots, d)$ and constant matrices $F_{i j}$ $(i=1,2 ; j=1,2, \ldots, d)$ quantify the multiple randomly occurring sector-like nonlinearities, the constant $\bar{\mu}$ accounts for infinite distributed delays, and the new Lyapunov-Krasovskii functional (18) stems from the discrete-time delay-fractioning idea.

\section{An ILlustrative Example}

Following [3], [17], we consider the SMC problem for an F-404 aircraft engine system. Setting the sampling time $T=1.2 \mathrm{~s}$, we obtain the following discretized nominal system matrix

$$
A=\left[\begin{array}{ccc}
0.2504 & 0 & 0.3919 \\
0.0570 & 0.6188 & -0.0616 \\
0.0502 & 0 & 0.1262
\end{array}\right] .
$$

In the F-404 aircraft engine model, $x_{k}^{1}$ and $x_{k}^{2}$ represent the horizontal position and $x_{k}^{3}$ is the altitude of the aircraft. The control inputs $u_{k}^{1}$ and $u_{k}^{2}$ are the engine thrust and flight path angle, respectively. The movement of the aircraft is affected by the wind that acts as a stochastic disturbance $\omega_{k}$. To this end, other parameters are given as:

$$
\begin{aligned}
A_{d} & =\left[\begin{array}{ccc}
0.03 & 0 & -0.01 \\
0.02 & 0.03 & 0 \\
0.04 & 0.05 & -0.01
\end{array}\right], B=\left[\begin{array}{cc}
0.1817 & 0.4286 \\
0.1597 & 0.793 \\
0.1138 & 0.0581
\end{array}\right], \\
C & =\left[\begin{array}{ccc}
0.03 & 0.015 & -0.01 \\
0.02 & 0.03 & 0 \\
0.02 & 0.025 & -0.01
\end{array}\right], H=\left[\begin{array}{c}
0.01 \\
0.02 \\
0.005
\end{array}\right] \\
D & =\left[\begin{array}{ccc}
0.025 & 0.01 & 0 \\
0 & -0.03 & 0 \\
0.04 & 0.035 & -0.01
\end{array}\right], H_{d}=\left[\begin{array}{c}
0.02 \\
0.03 \\
0
\end{array}\right], \\
E & =\left[\begin{array}{ccc}
0.015 & 0 & -0.01 \\
0.01 & 0.015 & 0 \\
0.02 & 0.025 & -0.01
\end{array}\right], N^{T}=\left[\begin{array}{c}
0.2 \\
0.1 \\
0
\end{array}\right]
\end{aligned}
$$

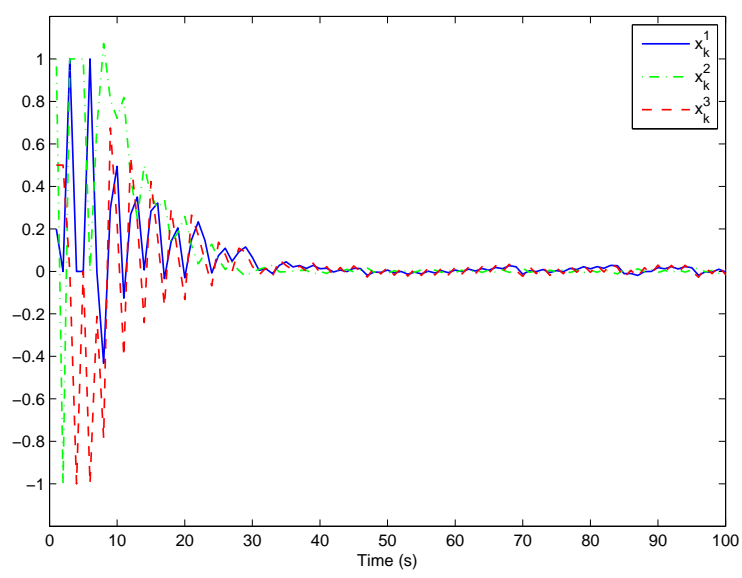

Fig. 1. The trajectory of state $x_{k}(\mathrm{~m})$

Let

$$
\begin{aligned}
& F=\sin (0.6 k), \\
& f\left(x_{k}\right)=\left[\begin{array}{ll}
0.4 \sin \left(x_{k}^{1} x_{k}^{3}\right) & 0.3 \sin \left(x_{k}^{2}\right)
\end{array}\right]^{T}, \\
& \sigma\left(x_{k}, x_{k-d_{k}}\right)=0.5 x_{k}+0.5 x_{k-d_{k}}, \\
& g_{1}\left(x_{k}\right)=0.5\left(F_{11}+F_{21}\right) x_{k}+0.5\left(F_{21}-F_{11}\right) \sin \left(x_{k}\right) x_{k}, \\
& g_{2}\left(x_{k}\right)=0.5\left(F_{12}+F_{22}\right) x_{k}+0.5\left(F_{22}-F_{12}\right) \cos \left(x_{k}\right) x_{k},
\end{aligned}
$$

where

$$
\begin{aligned}
& \sin \left(x_{k}\right):=\operatorname{diag}\left\{\sin \left(x_{k}^{1}\right), \sin \left(x_{k}^{2}\right), \sin \left(x_{k}^{3}\right)\right\}, \\
& \cos \left(x_{k}\right):=\operatorname{diag}\left\{\cos \left(x_{k}^{1}\right), \cos \left(x_{k}^{2}\right), \cos \left(x_{k}^{3}\right)\right\}, \\
& F_{11}=F_{12}=\operatorname{diag}\{0.4,0.5,0.8\}, \\
& F_{21}=F_{22}=\operatorname{diag}\{0.3,0.2,0.6\} .
\end{aligned}
$$

Set $\alpha=0.75, \beta=0.78, \gamma_{1}=0.86$ and $\gamma_{2}=0.82$. Assume that the time-varying delay $d_{k}$ satisfies $3 \leq d_{k} \leq 5$. Moreover, choosing the constants $\mu_{p}=2^{-3-p}$, we can easily see that the convergence condition (7) holds.

Setting $m=1$ and $\varrho=1.2 \times 10^{-4}$ in (18) and solving the minimization problem (28), we obtain

$$
P=\left[\begin{array}{ccc}
0.4676 & -0.0765 & -0.1145 \\
-0.0765 & 0.1880 & -0.0459 \\
-0.1145 & -0.0459 & 0.3346
\end{array}\right],
$$

and $\gamma=6.9607 \times 10^{-5}$ (hence the equality constraint in Eq. (17) is considered to be achieved). It follows from Theorem 2 that the desired SMC controller (32) can be described by all known parameters. The simulation results are shown in Figs. 1-3. Among them, Fig. 1 shows the response of system state by taking $\mu_{j}=\nu_{j}=0.001(j=1,2)$. The responses of sliding surface $s_{k}$ and control input $u_{k}$ are shown in Figs. 2-3, respectively. The simulation results have confirmed our theoretical results. It would be interesting to look into the possibility of carrying out real-time experiments on flight control systems with actual engines in the future.

\section{Conclusions}

The robust SMC problem for a class of discrete mixed time-delays stochastic system with ROUs and RONs has been 


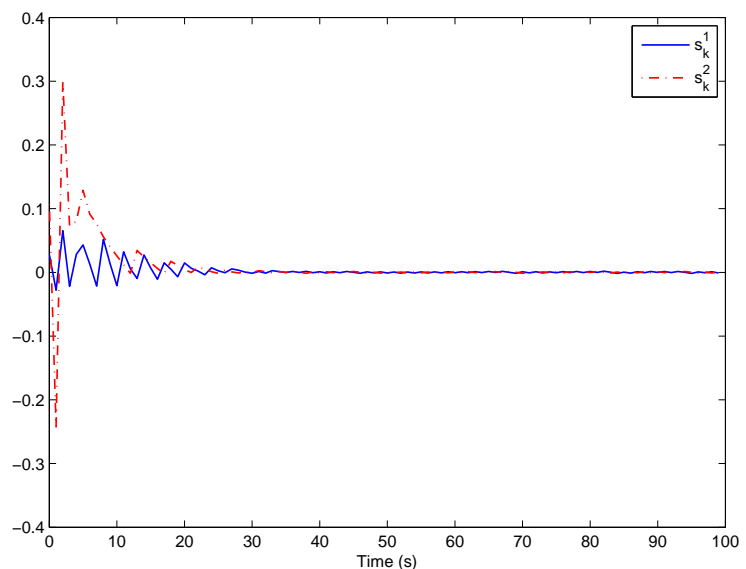

Fig. 2. The trajectory of sliding variable $s_{k}$

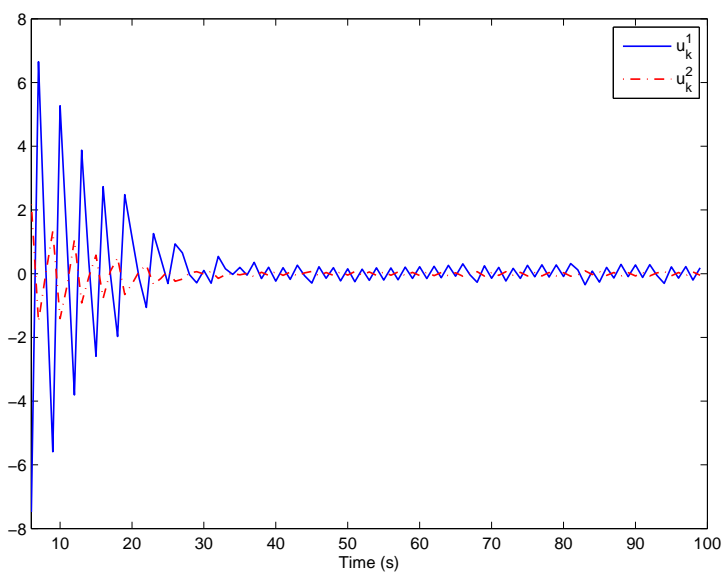

Fig. 3. The control signals $u_{k}^{1}(k N)$ and $u_{k}^{2}(\mathrm{deg})$

considered in this paper. By making use of delay-fractioning approach and constructing a new Lyapunov-Krasovskii functional, a sufficient condition has been derived to ensure the stability of the sliding mode dynamics, and an SMC law has been designed such that the state trajectory of the system starting from any initial state is globally driven onto the specified sliding surface. Further research topics include the SMC problem for networked control system with plant output delays and input delays.

\section{REFERENCES}

[1] K. Abidi, J. X. Xu and X. H. Yu, "On the discrete-time integral slidingmode control," IEEE Trans. Automat. Control, vol. 52, no. 4, pp. 709715, Apr. 2007.

[2] H. H. Choi, "LMI-based sliding surface design for integral sliding mode control of mismatched uncertain systems," IEEE Trans. Automat. Control, vol. 52, no. 4, pp. 736-742, Apr. 2007.

[3] R. W. Eustace, B. A. Woodyatt, G. L. Merrington and A. Runacres, "Fault signatures obtained from fault implant tests on an F404 engine," ASME Transactions Journal of Engineering for Gas Turbines and Power, vol. 116, no. 1, pp. 178-183, Jan. 1994.

[4] G. Foo and M. F. Rahman, "Sensorless sliding-mode MTPA control of an IPM synchronous motor drive using a sliding-mode observer and HF signal injection," IEEE Trans. Industrial Electronics, vol. 57, no. 4, pp. 1270-1278, Apr. 2010.
[5] W. B. Gao, Y. F. Wang and A. Homaifa, "Discrete-time variable structure control systems," IEEE Trans. Industrial Electronics, vol. 42, no. 2, pp. 117-122, Apr. 1995.

[6] R. Gupta, A. Ghosh and A. Joshi, "Multiband hysteresis modulation and switching characterization for sliding-mode-controlled cascaded multilevel inverter," IEEE Trans. Industrial Electronics, vol. 57, no. 7, pp. 2344-2353, Jul. 2010.

[7] L. R. Huang and X. R. Mao, "SMC design for robust $H_{\infty}$ control of uncertain stochastic delay systems," Automatica, vol. 46, no. 2, pp. 405412, Feb. 2010.

[8] S. Janardhanan and B. Bandyopadhyay, "Output feedback sliding-mode control for uncertain systems using fast output sampling technique," IEEE Trans. Industrial Electronics, vol. 53, no. 5, pp. 1677-1682, Oct. 2006

[9] H. K. Khalil, Nonlinear systems, Upper Saddle River, NJ: Prentice-Hall, 1996.

[10] N. O. Lai, C. Edwards and S. K. Spurgeon, "On output tracking using dynamic output feedback discrete-time sliding-mode controllers," IEEE Trans. Automat. Control, vol. 52, no. 10, pp. 1975-1981, Oct. 2007.

[11] Y. G. Niu, D. W. C. Ho and J. Lam, "Robust integral sliding mode control for uncertain stochastic systems with time-varying delay," Automatica, vol. 41, no. 5, pp. 873-880, May. 2005.

[12] T. Orlowska-Kowalska, M. Kamiński and K. Szabat, "Implementation of a sliding-mode controller with an integral function and fuzzy gain value for the electrical drive with an elastic joint," IEEE Trans. Industrial Electronics, vol. 57, no. 4, pp. 1309-1317, Apr. 2010.

[13] D. Peaucelle, D. Arzelier, D. Henrion and F. Gouaisbaut, "Quadratic separation for feedback connection of an uncertain matrix and an implicit linear transformation," Automatica, vol. 43, no. 5, pp. 795-804, May. 2007.

[14] C. Pukdeboon, A. S. I. Zinober and M.-W. L. Thein, "Quasi-continuous higher order sliding-mode controllers for spacecraft-attitude-tracking maneuvers," IEEE Trans. Industrial Electronics, vol. 57, no. 4, pp. 14361444, Apr. 2010.

[15] P. Shi, Y. Q. Xia, G. P. Liu and D. Rees, "On designing of sliding-mode control for stochastic jump systems," IEEE Trans. Automat. Control, vol. 51, no. 1, pp. 97-103, Jan. 2006.

[16] E. Vidal-Idiarte, C. E. Carrejo, J. Calvente and L. Martínez-Salamero, "Two-loop digital sliding mode control of DC-DC power converters based on predictive interpolation," IEEE Trans. on Industrial Electronics, vol. 58, no. 6, pp. 2491-2501, Jun. 2011.

[17] Z. D. Wang, Y. R. Liu and X. H. Liu, " $H_{\infty}$ filtering for uncertain stochastic time-delay systems with sector-bounded nonlinearities," $\mathrm{Au}$ tomatica, vol. 44, no. 5, pp. 1268-1277, May. 2008.

[18] Z. D. Wang, Y. Wang and Y. R. Liu, "Global synchronization for discrete-time stochastic complex networks with randomly occurred nonlinearities and mixed time delays," IEEE Trans. Neural Networks, vol. 21, no. 1, pp. 11-25, Jan. 2010.

[19] L. G. Wu and D. W. C. Ho, "Sliding mode control of singular stochastic hybrid systems," Automatica, Vol. 46, No. 4, pp. 779-783, Apr. 2010.

[20] Y. Q. Xia, G. P. Liu, P. Shi, J. Chen, D. Rees and J. Liang, "Sliding mode control of uncertain linear discrete time systems with input delays," IET Control Theory Appl., vol. 1, no. 4, pp. 1169-1175, Apr. 2007.

[21] M. Yan and Y. Shi, "Robust discrete-time sliding mode control for uncertain systems with time-varying state delay," IET Control Theory Appl., vol. 2, no. 8, pp. 662-674, Aug. 2008.

[22] X. H. Yu and O. Kaynak, "Sliding-mode control with soft computing: a survey," IEEE Trans. Industrial Electronics, vol. 56, no. 9, pp. 32753285, Sep. 2009.

[23] J. H. Zhang and Y. Q. Xia, "Design of static output feedback sliding mode control for uncertain linear systems," IEEE Trans. Industrial Electronics, vol. 57, no. 6, pp. 2161-2170, Jun. 2010. 


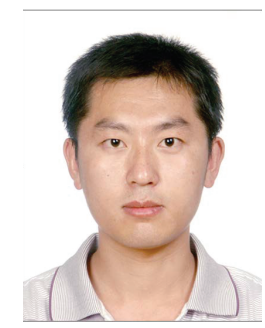

Jun Hu received his B.S. degree in Information and Computation Science from Harbin University of Science and Technology, Harbin, China, in 2006, and his M.S. degree in Applied Mathematics from Harbin University of Science and Technology, Harbin, China, in 2009. He is currently pursuing the $\mathrm{Ph} . \mathrm{D}$. degree in the School of Astronautics, Harbin Institute of Technology, Harbin, China. He is now a Visiting PhD Student in the Department of Information Systems and Computing, Brunel University, U.K. His research interests include nonlinear control and filtering, stochastic systems. He is a very active reviewer for many international journals.

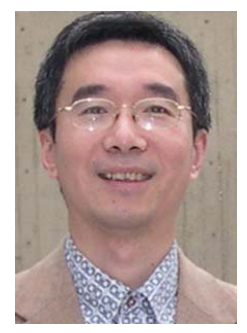

Zidong Wang (SM'03) was born in Jiangsu, China, in 1966. He received the B.Sc. degree in mathematics in 1986 from Suzhou University, Suzhou, China, and the M.Sc. degree in applied mathematics in 1990 and the Ph.D. degree in electrical engineering in 1994, both from Nanjing University of Science and Technology, Nanjing, China.

$\mathrm{He}$ is currently Professor of Dynamical Systems and Computing in the Department of Information Systems and Computing, Brunel University, U.K. From 1990 to 2002, he held teaching and research appointments in universities in China, Germany and the UK. Prof. Wang's research interests include dynamical systems, signal processing, bioinformatics, control theory and applications. He has published more than 100 papers in refereed international journals. He is a holder of the Alexander von Humboldt Research Fellowship of Germany, the JSPS Research Fellowship of Japan, William Mong Visiting Research Fellowship of Hong Kong.

Prof. Wang serves as an Associate Editor for 12 international journals, including IEEE Transactions on Automatic Control, IEEE Transactions on Control Systems Technology, IEEE Transactions on Neural Networks, IEEE Transactions on Signal Processing, and IEEE Transactions on Systems, Man, and Cybernetics - Part C. He is a Senior Member of the IEEE, a Fellow of the Royal Statistical Society and a member of program committee for many international conferences.

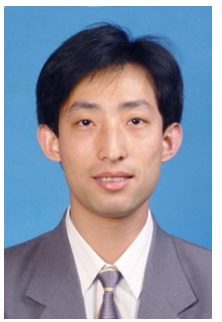

Huijun Gao (SM'09) was born in Heilongjiang Province, China, in 1976. He received the M.S. degree in electrical engineering from Shenyang University of Technology, Shengyang, China, in 2001, and the Ph.D. degree in control science and engineering from Harbin Institute of Technology, Harbin, China, in 2005 .

Dr. Gao is now a Professor at Harbin Institute of Technology, China. His research interests include network-based control, robust control/filter theory, model reduction, time-delay systems, and multidimensional systems, and their applications. He has published more than 80 papers in refereed international journals.

Dr. Gao is an Associate Editor or member of editorial board for several journals, such as Automatica, IEEE Transactions on Systems, Man, and Cybernetics - Part B, IEEE Transactions on Industrial Electronics, IEEE Transactions on Fuzzy Systems, IEEE Transactions on Circuits and Systems.
Lampros K. Stergioulas is Reader in the Department of Information Systems and Computing at Brunel University, United Kingdom. A qualified chartered engineer, he has studied informatics and physics at the University of Athens, and received an M.Sc. and a Ph.D. in electrical engineering from the University of Liverpool, specializing in information engineering and communications. Dr. Stergioulas has published over 150 papers in journals and international conferences. He has been a principal investigator in numerous EU and UK projects, and coordinator of three multi-site European projects. His research interests include signal processing, intelligent information processing, technologyenhanced learning, educational and health information systems, and humancentered computing. 